Methods Annual check-ups done every year from 2012 to 2016 in two medium-sized enterprises allow me to conduct a retrospective study on records of more than 300 workers every year. Then I compare the outcomes between the workers of the same enterprise and between the workers of the two companies in terms of prevalence, and of types of illnesses and occupational risk factors related to them.

Results and discussion Annual check-up is legal prescription for every enterprise in Senegal and more than 90\% workers undergo this medical survey done by an occupational physician. The company profile is established and allow us to see interesting differences and or similarities.

Conclusion Annual check-up is a powerful tool and a good opportunity for both occupational hazards prevention and health promotion for the wellbeing of workers and for the performance of enterprises.

\section{MINI SYMPOSIUM FOR ASEAN DIAGNOSTIC CRITERIA FOR OCCUPATIONAL DISEASES}

Adul Bandhukul. Nopparat Rajchathani Hospital, Bangkok, Thailand

\subsection{6/oemed-2018-ICOHabstracts. 1112}

Introduction The Association of South East Asian Nations (ASEAN) was formed in August 1967 as a regional grouping to promote economic growth and socio-cultural progress. The founder members were Indonesia, Malaysia, Philippines, Singapore and Thailand. It expanded to a group of ten Asian countries, with the further inclusion of Brunei, Cambodia, Laos, Myanmar and Vietnam. By the end of 2015, it was intended that a closer ASEAN community linkage would be fully established through economic integration, cooperation and harmonisation which we can foresee the migration of workforces between ten ASEAN countries. With the formation of ASEAN community, there was also a recognition that there should be harmonisation of occupational health services, especially the standards and procedures including diagnostic criteria for occupational diseases.

Methods There were 5 meeting on these ASEAN Diagnostic Criteria for Occupational Diseases (ADCOD).

Results The ADCOD that was finished were Occupational Asthma, Occupational contact dermatitis, Heat stress, Occupational Noise Induced Hearing Loss, Chronic Silicosis, Occupational Pesticide Poisoning: Organophosphate and Carbamate Poisoning, Disease caused by lead and its Toxic Compounds: Inorganic Lead Poisoning, Carpal Tunnel Syndrome and Asbestosis. The criteria that was recently discussed in the 5th meeting are Diseases caused by Arsenic and its toxic compounds, Poisoning due to Trichloroethylene, Occupational diseases due to Vibration, Occupational Hepatitis B viral disease, Rotator cuff tendinopathies, Decompression illness and Mercury poisoning. We will discuss further how to execute these criteria to the ASEAN country. We also discuss about the ASEAN Network on Occupational Diseases Reporting-ANODE in which we will have the statistic of Occupational Diseases in each country in real time. This is very useful for preventing diseases.

Conclusion After we finished the meeting in June. We will have 16 ADCOD. This is very helpful for the Occupational Health system in ASEAN for we will have the criteria for diagnosis in the same in all ASEAN country. The diagnosis will be accurate for monitor the occupational health system. If there is a disease there will be some mistake in the system that need to correct. We will soon have the Network for Occupational Diseases reporting that will be very useful for ASEAN countries.

\section{OCCUPATIONAL METHANOL EXPOSURE IS NOT RELATED TO CANCER MORTALITY: 12-YEAR FOLLOW-UP STUDY FOR TWENTY-FIVE THOUSAND MALE WORKERS IN KOREA}

${ }^{1}$ Gab-Sik Shin*, ${ }^{2}$ Yeon-Soon Ahn* ${ }^{2}{ }^{2}$ eon-Soo An, ${ }^{2}$ Hyeong-Do Kim. ${ }^{1}$ Departments of Occupational and Environmental Medicine, Dongguk University Gyeongju Hospital, Gyeongju, Republic of Korea; '2Department of Occupational and Environmental Medicine, Dongguk University Ilsan Hospital, 29 Dongguk-no, Ilsandong-gu, Goyang 410-773, Republic of Korea

\subsection{6/oemed-2018-ICOHabstracts.1113}

Introduction An International Agency for Research on Cancer (IARC) Advisory Group recommended priorities for IARC Monographs during 2015-2019 in Lyon on April, 2014. They recommended methanol as medium priority of review. The background was three animal carcinogenicity studies. However there were no available studies of cancer in humans and has not been previously evaluated by IARC. So we analysed the cancer mortality of methanol exposed male workers in Korea. Methods A cohort was comprised of 25218 methanol workers working between January 1, 2000, and December 31, 2004. These cohort members were matched with the mortality data of the Korean National Statistical Office to follow-up for cancer mortality between 2000 and 2011. Standardised Mortality Ratios (SMRs) of methanol exposed workers with reference to Korean men were calculated. Also controlling age, calendar year and other carcinogen exposure including hepatitis B and C, the Adjusted Hazard Ratios (AHRs) of workers categorised by the 2 groups of methanol exposure level (10\% to 50\%, over $50 \%$ of TLV) with reference to workers with less than $10 \%$ were calculated.

Result There were no significantly increased SMRs. But, significantly decreased SMRs were observed overall cancer $(\mathrm{SMR}=0.70$, 95\% CI: 0.58 to 0.84$)$ and liver cancer $(\mathrm{SMR}=0.68,95 \% \mathrm{CI}$ : 0.47 to 0.94$)$. There were no significantly increased or decreased AHRs of cancer mortalities in workers exposed to methanol with $10 \%-50 \%$ of TLV and over $50 \%$ of TLV compared to workers with less than $10 \%$ of TLV.

Discussion In this study short follow-up periods and healthy worker effect (HWE) may hamper observation for increasing cancer mortality of methanol exposed workers comparing to that of Korean male. However based on no increased AHRs of workers with relatively higher level of methanol exposure, methanol might not be related to cancer development considering metabolic pathway different from ethanol. Continuous follow-up to overcome HWE and cancer morbidity study are needed to confirm this study result.

Acknowledgement This work was supported by Korea Environment Industry and Technology Institute (KEITI) through The Chemical Accident Prevention Technology Development Project funded by Korea Ministry of Environment (MOE) (2017001970001). 